Tamoxifen can prevent breast cancer in high-risk women, although most studies have focused on the prevention of cancers during the active treatment phase. The International Breast Cancer Intervention Study I (IBIS-I) investigators now report that tamoxifen taken for 5 years continues to prevent breast cancers for up to 5 additional years after stopping treatment. Moreover, most side effects disappear once tamoxifen therapy is stopped.

The IBIS-I trial randomized 7,154 women at increased risk of breast cancer to receive either tamoxifen or placebo for 5 years. After an initial median follow-up of 49.6 months, tamoxifen reduced the risk of breast cancer by $32 \%$. The investigators now find that, with a median follow-up of 8 years, tamoxifen continues to provide a significant reduction (27\%) in the overall incidence of breast cancers, and this effect was observed for up to 10 years after randomization. The reduction was greatest for oestrogen receptor-positive tumours, which were $26 \%$ lower in the tamoxifen group during tamoxifen treatment and $44 \%$ lower in subsequent years.

The investigators also found that side effects, specifically deep-vein thrombosis, pulmonary embolism and endometrial cancer, were higher in the tamoxifen group during active treatment but not once tamoxifen was stopped, thus improving the risk:benefit ratio of tamoxifen as a breast cancer preventative agent.

ORIGINAL RESEARCH PAPER Cuzick, J. et al. Long-term results of tamoxifen prophylaxis for breast cancer - 96-month follow-up of the randomized IBIS-I trial. J. Natl Cancer Inst. 99, 272-282 (2007)

\title{
NEW LUNG CANCER THERAPIES
}

Two new therapies for non-small-cell lung cancer (NSCLC) have entered phase III clinical trials.

The first patient has been enrolled in a randomized, doubleblind, placebo-controlled phase III trial of the BLP25 liposome vaccine (L-BLP25, also known as Stimuvax) that is expected to enroll more than 1,300 patients with stage III NSCLC. L-BLP25 is a vaccine designed to stimulate the immune system by targeting the protein mucin 1 (MUC1), which is widely expressed on cancer cells. A phase Ilb trial with L-BLP25 found that median survival was 30.6 months versus 13.3 months in the control group for patients with stage IIlb locoregional disease.

A new placebo-controlled phase III trial of vandetanib (also known as ZD6474 or Zactima) in at least 508 patients with NSCLC was also announced. Vandetanib inhibits the epidermal growth factor receptor (EGFR) and rearranged during transfection (RET) tyrosine kinases in tumour cells, and also inhibits angiogenesis by targeting vascular endothelial growth factor receptor (VEGFR). Vandetanib showed anti-tumour activity in phase II trials in NSCLC, and three other phase III trials of vandetanib in NSCLC are ongoing.

As approximately $80 \%$ of patients with lung cancer have NSCLC, and only about $15 \%$ of these survive after 5 years, these therapies could lead to a better outcome.

WEB SITES http://www.biomira.com; http://www.astrazeneca-us.com 